Int. J. Odontostomat.,

13(1):40-45, 2019.

\title{
Contribución al Estudio Descriptivo del Foramen y Canal Palatino Mayor
}

\author{
Contribution to the Descriptive Study of the Foramen and Greater Palatine Canal
}

\author{
Andrés Salcedo'; Claudia Araya²; Juan Silva²; Nicolas Barraza² \& Alexis Latín²
}

SALCEDO, A.; ARAYA, C.; SILVA, J.; BARRAZA, N. \& LATíN, A. Contribución al estudio descriptivo del foramen y canal palatino mayor. Int. J. Odontostomat., 13(1):40-45, 2019.

RESUMEN: El foramen y canal palatino mayor (FPM y CPM) comunican boca con fosa pterigopalatina. El conocimiento adecuado de su morfología, permite el abordaje anestésico del nervio maxilar. En el vivo, el FPM está recubierto por una mucosa gruesa, debido a esto los puntos de referencia óseos y dentarios son importantes para ubicar el sitio de punción. Se ha descrito gran variabilidad en cuanto a la etnia, posición, forma, diámetros, longitudes y permeabilidad. Este estudio tuvo como objetivo describir éstas características en cráneos de adultos chilenos. Se utilizaron 31 cráneos de ambos sexos. En los paladares se determinó; forma, largo, profundidad y ancho. En los FPM se consideró su forma, diámetros y localización. En los CPM se registró su permeabilidad y su coincidencia con la forma del FPM. Los registros se realizaron con cámara digital, compás de precisión, caliper digital, compas tridimensional de Korkhaus y sonda metálica. Los resultados muestran un predominio de la forma cuadrada del paladar por sobre las formas triangular y redondeada. Las mediciones de su largo, ancho y profundidad indican diferencias por sexo y por etnia. La forma del FPM no muestra diferencia por sexo, primando la forma ovalada por sobre la fusiforme y la redondeada. La posición de este mismo foramen tampoco muestra diferencias sexuales, primando la posición frente al tercer molar superior, seguida por la posición frente al espacio entre segundo y tercer molar superior y por último frente al 2do molar superior. Las dimensiones del FPM son mayores en individuos masculinos. Los CPM se observaron en su totalidad permeables y los FPM no siempre coincidieron en forma con la sección transversal del CPM. Estos resultados y su comparación con la literatura indican variaciones importantes, lo que impide establecer directrices objetivas a la técnica anestésica que utiliza esta vía anatómica.

PALABRAS CLAVE: foramen palatino mayor, canal palatino mayor, fosa pterigopalatina, hueso maxilar, hueso palatino, técnica anestésica de Carrea.

\section{INTRODUCCIÓN}

El canal palatino mayor (CPM) es un conducto óseo que representa una vía de comunicación entre la fosa pterigopalatina (FPP) y la cavidad oral, está formado por la unión de 2 semicanales excavados tanto en la tuberosidad del hueso maxilar como en la lámina vertical de hueso palatino. Presenta su origen en el ángulo inferior de la FPP y termina en los ángulos posteriores de la bóveda palatina, en el foramen palatino mayor (FPM). Está destinado a permitir el tránsito del nervio palatino mayor y la arteria palatina descendente. A su vez, el FPM es un orificio de forma ovalada o redondeada situado en el ángulo diedro que forman la porción horizontal de palatino con la cara interna del margen alveolar, cerca del margen posterior del pala- dar óseo (a la altura del 3er molar superior), en una fosa característica que es posible palpar y/u observar en forma clínica. También está formado por los huesos maxilar y palatino, y se considera el extremo terminal o inferior del CPM (Cruveilhier, 1851; Sappey, 1874; Beaunis \& Bouchard, 1878; Poirier et al., 1906; Carrea, 1921; Testut \& Latarjet, 1965; Aprile et al., 1967; Triantafilo, 1968; Ries-Centeno, 1973; Alvarado et al., 1982; Velayos, 2001; Latarjet \& Ruiz Liard, 2004; Dauber, 2007; Tomaszewska et al., 2015).

El conocimiento específico y detallado de la conformación, ubicación, dirección, longitud, relaciones, contenido y diferencias etarias, étnicas y sexuales,

\footnotetext{
${ }^{1}$ Facultad de Odontología, Universidad San Sebastian, Chile.

${ }^{2}$ Facultad de Medicina, Universidad San Sebastian, Chile.
} 
permite abordar con éxito el FPM y el CPM, para procedimientos quirúrgicos odontológicos extensos 0 cuando otras técnicas anestésicas no puedan ser utilizadas por procesos infecciosos y/o inflamatorios en el sitio de punción, permitiendo realizar un correcto bloqueo anestésico local y troncular al nervio maxilar, ramo del nervio trigémino (Carrea; Alvarado et al.; Malamed \& Trieger, 1983; Malamed, 2006; Howard-Swirzinski et al., 2010; Lopes et al., 2011; Nimigean et al., 2013; Sheikhi et al., 2013; llayperuma et al., 2014; Tomaszewska et al., 2014; RapadoGonzález et al., 2015).

Con respecto a esto, el Dr. Juan Ubaldo Carrea señala en su célebre trabajo de investigación de 1925, "que la técnica anestésica del nervio maxilar superior, tomando por vía el conducto palatino posterior representa el procedimiento más seguro, más simple, mas inocuo y más eficaz para lograr el bloqueo anestésico de la región inervada por dicho nervio".

El FPM está formado por una escotadura observada en el proceso palatino de maxilar y completada por otra escotadura, observada en el hueso palatino. Se ha descrito predominantemente de forma ovalada, pero también se ha observado fusiforme y redondeada. De 3,0 a 7,35 mm de diámetro antero-posterior por 2,3 a 4,0 mm de diámetro transversal. En su margen posterior se ha descrito que es posible observar una cresta ósea que lo separa de los forámenes palatinos menores (Sicher \& Tandler, 1930; Aprile et al.; Ilayperuma et al.; Rapado-González et al.; Sarilita \& Soames, 2015). Estos mismos autores describen diferencias étnicas y sexuales importantes.

Debido a que el FPM se encuentra recubierto por una mucosa gruesa que oculta su posición al examen clínico, los puntos de referencia óseos y dentarios son fundamentales para encontrar esta estructura anatómica que permite ubicar el FPM, el CPM, la FPP y el tronco del nervio maxilar (Rapado-González et al.).

El FPM se ubica a $10 \mathrm{~mm}$ por delante del gancho pterigoideo y a 5 a $6 \mathrm{~mm}$ del límite de separación entre paladar duro y paladar blando, medial a la cara palatina del 3er molar superior o ligeramente detrás de la cara distal del 2 do molar superior. Pero se describen grandes variaciones según edad y características individuales (Aprile et al.; Ries-Centeno).

En cráneos secos de individuos tailandeses se ha observado una distancia media entre el FPM y la línea media de 16,2 +1,3 mm, distancia entre FPM y el margen posterior del paladar duro de 2,1+1,3 mm y entre FPM a la cresta alveolar una distancia promedio de 13,8 + 1,3 mm (Methathrathip et al., 2005).

En un gran número de cráneos provenientes de Norteamérica, Europa y África, se logró determinar que el FPM se ubica mayoritariamente frente a la mitad anterior del 3 er molar superior (50,63 \% de los casos), seguido de una ubicación frente a la mitad posterior del 2 do molar superior $(39,87 \%$ de los casos y finalmente en menor proporción, frente a la mitad posterior del 3er molar superior (9,49\% de los casos) (Malamed \& Trieger).

Se ha estimado que el largo del CPM correspondería a $14 \mathrm{~mm}$ y presentaría una dirección oblicua hacia arriba en un rango que se presentaría entre los $20^{\circ}$ a $70^{\circ}$ de inclinación con respecto al paladar duro (unos $45^{\circ}$ en promedio) y casi paralela al plano sagital. Así mismo, se ha observado que la distancia desde el FPM al foramen redondo (FR) sería de unos 28 a 33 $\mathrm{mm}$. También se ha observado en un 5 a un $15 \%$, que existirían conductos con obstrucción en su trayecto (Ries-Centeno; Alvarado et al.; Velayos; Methathrathip et al.; Malamed).

Debido a que las descripciones anatómicas publicadas acerca del FPM y el CPM no han sido lo suficientemente consistentes y existen declaradas discrepancias en los textos de anatomía clásicos (Ajmani, 1994), el objetivo de este trabajo es determinar, en una muestra de cráneos chilenos de ambos sexos las características morfológicas del FPM y del CPM que son de gran interés para la anatomía humana normal, la odontología, la cirugía maxilo-facial, la otorrinolaringología y la anestesiología.

\section{MATERIAL Y MÉTODO}

Se utilizaron 31 cráneos secos de individuos adultos chilenos de los cuales 19 eran de sexo masculino $(61,29 \%$ de la muestra) y 12 de sexo femenino $(38,71 \%$ de la muestra). De estos cráneos, 3 eran dentados (9,68 \% de la muestra), 15 eran desdentados parciales $(48,38 \%$ de la muestra) y 13 eran edéntulos (41,94\% de la muestra). En este material óseo se observaron y analizaron 62 FPM y 62 CPM. Este material óseo pertenece a la osteoteca de la Facultad de Medicina de la Universidad San Sebastian, Chile. 
De los paladares óseos, se determinó su forma (redondeado, cuadrado y triangular), su largo, su ancho intermolar (distancia entre las tablas óseas internas de la región entre los 2 dos y 3eros molares superiores), su ancho intercanino (distancia entre las tablas óseas internas de la región de los caninos superiores) y su profundidad. De los FPM analizados, se consideró su forma (ovalado, redondeado y fisurado), su tamaño (diámetro antero-posterior y diámetro transversal), su localización (en virtud de su relación con la línea media, el margen posterior del paladar óseo, el foramen interincisivo, el fondo de la fosa pterigopalatina, el surco hamular, el plano sagital y los segundos y terceros molares superiores). De los CPM analizados, se registró su permeabilidad y su coincidencia con la forma del FPM. La Figura 1 esquematiza estas medidas.

Los cráneos se fotografiaron con cámara digital marca Canon` modelo PowerShot@ D30. Luego se procedió a su medición con compás de precisión marca Staedtler ( , modelo Mars 552, caliper digital marca Mitutoyo (C) serie 500 modelo 144 y compas tridimensional de Korkhaus. La permeabilidad de los



Fig. 1. Esquema de paladar óseo donde se observan las principales mediciones realizadas en la estudio. 1.- Distancia entre tabla ósea interna de la región interincisiva y la espina nasal posterior. 2.- Ancho intermolar. 3.- Ancho interincisivo. 4.- Largo foramen palatino mayor. 5.- Ancho foramen palatino mayor. 6.- Distancia foramen palatino mayor y la línea media. 7.- Distancia entre foramen palatino mayor y el borde palatino posterior. 8.- Distancia entre foramen palatino mayor y foramen incisivo. 9.- Plano sagital de referencia del foramen palatino mayor,en este caso el foramen se encuentra a la altura del canino. 10.- Distancia entre el foramen palatino mayor y el surco hamular $(\mathrm{SH})$.
CPM se realizó con sonda metálica. Todas estas mediciones fueron tomadas 3 veces y el valor registrado corresponde al promedio simple. Los resultados se registraron para su análisis estadístico descriptivo en formulario ad hoc.

\section{RESULTADOS Y DISCUSIÓN}

La Tabla I, muestra los resultados obtenidos en relación a la forma de paladar. La forma cuadrada (que incluye a los estrictamente cuadrados y a los cuadrados con una región redondeada en la región intercanina) correspondió a la mayoría de los casos, seguidos por la forma triangular y finalmente los redondeados.

La Tabla II, resume los resultados de las dimensiones; largo, ancho y profundidad del paladar óseo. En forma general se puede observar una diferencia determinada por sexo, siendo los femeninos de menores dimensiones que los masculinos. Estos resultados son algo distintos a los observados por Sarilita \& Soames, quienes describen en cráneo indios, paladares más largos, más profundos, pero más angostos.

La Tabla III, reseña los resultados de la forma del FPM, los resultados diferenciados por sexo indican una similitud total, primando la forma ovalada frente a la fusiforme y la redondeada, estos resultados son concordantes con aquellos descritos en la literatura (Aprile et al.; Lopes et al.; Sarilita \& Soames), con respecto a la forma que prima (ovoidales frente a las otras formas), pero ésta distribución es distinta con aquellos observados en individuos de Europa, de Tailandia y de Sri Lanka (Methathrathip et al.; Nimigean et al.; Ilayperuma et al.). Esta diferencia puede deberse a que en estos estudios se pudo haber incluido en los ovalados, los fusiformes (debido a que en ambos se observa una forma alargada antero-posterior).

También son coincidentes entre los sexos, la posición del FPM, primando la posición frente al tercer molar superior, seguida por la posición frente al espacio entre segundo y tercer molar superior. Finalmente, con una diferencia importante, la posición frente al 2do molar superior. Estos resultados son algo discordantes con lo observado por Aprile et al., Riescenteno y Nimigean et al., pero son bastantes coincidentes con lo descrito por Malamed \& Trieger.

La Tabla IV, resume los resultados obtenidos con respecto a las dimensiones observadas en los FPM 
Tabla I. Resultados de la forma de paladar. Los "cuadrados" resumen; aquellos estrictamente de forma cuadrada $(16,13 \%$ de la muestra) y aquellos que presentan una forma más bien redondeada en la región intercanina (35,48\% de la muestra).

Forma Paladar

1<smiles>[IH2]</smiles>

2<smiles>[101In]</smiles>

Redondo $\quad 5(16,13 \%)$

Cuadrado

Triangular
$17(51,61 \%)$

$10(32,26 \%)$ de la muestra. Prevalece el tamaño mayor de los individuos masculinos en relación a los femeninos, tanto en largo como en ancho, estos resultados tienden a coincidir con lo evidenciado por Nimigean et al.

Esta misma tabla también nos muestra las medidas de la posición del FPM en los individuos de la muestra con respecto a la línea media (LM) y el margen posterior del paladar (MPP), siendo estos resultados concordantes para la primera medida pero discordantes para la segunda con aquello indicado por Malamed \& Trieger y Methathrathip et al.

Así también, la Tabla IV indica los resultados para las mediciones entre el FPM y el fondo de la fosa pterigoidea (FP), este resultado es algo concordante con lo indicado por Alvarado et al.

Por otra parte nuestros resultados para la distancia entre el FPM y el surco hamular $(\mathrm{SH})$ no es semejante con lo descrito por Malamed \& Trieger, así mientras el rango de estos autores varia de 5 y hasta los $20,5 \mathrm{~mm}$, en el caso nuestro esta medida varia de 2,32 a $12,21 \mathrm{~mm}$.

Tabla II. Dimensiones del paladar. Largo: corresponde a la distancia entre la tabla ósea interna de la región interincisiva y la espina nasal posterior. Ancho intermolar: corresponde a la distancia entre tablas óseas internas de la región entre 2 do y 3 er molar derecho e izquierdo. Ancho intercanino: es la distancia entre tablas óseas internas de la región del canino derecho e izquierdo. Profundidad: corresponde a la distancia entre el borde más saliente del reborde óseo molar y la superficie más profunda del paladar. Todas las cifras se encuentran en $\mathrm{mm}$.

\begin{tabular}{lcccc}
\hline & Largo & $\begin{array}{c}\text { Ancho } \\
\text { intermolar }\end{array}$ & $\begin{array}{c}\text { Ancho } \\
\text { intercanino }\end{array}$ & Profundidad \\
\hline Muestra & 44,37 & 36,57 & 24,15 & 11,31 \\
Femenino & 43,25 & 35,93 & 23,05 & 10,21 \\
Masculino & 45,08 & 36,97 & 24,84 & 12,00 \\
\hline
\end{tabular}

Tabla III. Forma FPM: resultados de la forma geométrica del foramen palatino mayor. Posición FPM: posición del foramen palatino mayor en relación a molares superiores ("2M" corresponde al segundo molar superior y "3M" al 3er molar superior).

\begin{tabular}{|c|c|c|c|}
\hline \multicolumn{2}{|c|}{ Forma FPM } & \multicolumn{2}{|c|}{ Posición FPM } \\
\hline \multicolumn{4}{|c|}{ Lado Derecho } \\
\hline Fusiformes & $8(25,81 \%)$ & Frente $2 \mathrm{M}$ & $2(6,45 \%)$ \\
\hline Ovalado & $14(45,16 \%)$ & $2 M-3 M$ & $13(41,94 \%)$ \\
\hline Redondo & $9(29,03 \%)$ & Frente $3 \mathrm{M}$ & $16(51,61 \%$ \\
\hline \multicolumn{4}{|c|}{ Lado Izquierdo } \\
\hline Fusiformes & $8(25,81 \%)$ & Frente $2 \mathrm{M}$ & $2(6,45 \%)$ \\
\hline Ovalado & $14(45,16 \%)$ & $2 M-3 M$ & $13(41,94 \%)$ \\
\hline Redondo & $9(29,03 \%)$ & Frente $3 \mathrm{M}$ & $16(51,61 \%$ \\
\hline
\end{tabular}


Tabla IV. Largo FPM: distancia máxima entre el borde anterior y el borde posterior del foramen palatino mayor. Ancho FPM: distancia máxima entre el borde medial y el borde lateral del foramen palatino mayor. Distancia FPM/LM: distancia medida entre el foramen palatino mayor y la línea media. Distancia FPM/BPP: distancia entre el foramen palatino mayor y el borde posterior del paladar óseo. Distancia FPM/FI: distancia entre el foramen palatino mayor y el foramen incisivo. Distancia FPM/FP: distancia entre el foramen palatino mayor y el fondo de la fosa pterigoidea. Distancia FPM/SH: distancia entre el foramen palatino mayor y el surco hamular. Todas las cifras se encuentran en $\mathrm{mm}$.

\begin{tabular}{|c|c|c|c|c|c|c|c|}
\hline & Largo FPM & Ancho FPM & $\begin{array}{l}\text { Distancia } \\
\text { FPM/LM }\end{array}$ & $\begin{array}{l}\text { Distancia } \\
\text { FPM/BPP }\end{array}$ & $\begin{array}{c}\text { Distancia } \\
\text { FPM/FI }\end{array}$ & $\begin{array}{l}\text { Distancia } \\
\text { FPM/FFP }\end{array}$ & $\begin{array}{c}\text { Distancia } \\
\text { FPM/SH }\end{array}$ \\
\hline \multicolumn{8}{|c|}{ Lado Derecho } \\
\hline Muestra & 5,56 & 3,55 & 15,99 & 4,90 & 35,80 & 9,59 & 7,94 \\
\hline Femenino & 4,99 & 3,35 & 15,39 & 4,56 & 35,62 & 9,46 & 7,49 \\
\hline Masculino & 5,92 & 3,68 & 16,47 & 5,12 & 35,91 & 9,67 & 8,23 \\
\hline \multicolumn{8}{|c|}{ Lado Izquierdo } \\
\hline Muestra & 5,42 & 3,45 & 15,64 & 4,82 & 35,81 & 9,82 & 8,35 \\
\hline Femenino & 5,03 & 3,15 & 14,69 & 4,61 & 35,75 & 9,47 & 7,56 \\
\hline Masculino & 5,66 & 3,64 & 16,23 & 4,95 & 35,85 & 10,03 & 8,85 \\
\hline
\end{tabular}

La Tabla V, revela que el FPM coincide en el plano sagital determinado por el canino prácticamente la misma cantidad de veces que se puede observar en el plano sagital determinado por el 1er premolar superior (aproximadamente en el $47 \%$ de los casos para cada uno), mientras que solo coincide con el espacio entre canino y primer molar superior, la menor cantidad de las ocasiones.

La misma tabla nos indica que la totalidad de los CPM fueron permeables a la sonda metálica, semejante a lo descrito por Malamed \& Trieger.
Finalmente pudimos percatarnos que la forma del FPM (fusiforme, ovalada, redonda) no siempre coincide con la sección transversal del CPM.

Estos resultados y su comparación con lo descrito en la literatura da cuenta de variaciones importantes con respecto a la morfología tanto del FPM y del CPM, lo que ha impedido dar adecuadas directrices a los profesionales de la salud que requieren anestesiar troncularmente al nervio maxilar.

Tabla V. Rel Sag FPM/PD: coincidencia de la posición del foramen palatino mayor, en un plano sagital; coincide con el plano determinado por el canino superior o con el espacio entre canino y primer premolar superior o con el primer premolar superior. Permeabilidad CPM: resultados de la exploración de la permeabilidad del conducto palatino mayor. Relación FPM/CPM: relación entre la forma del foramen palatino mayor y la forma de la sección transversal del conducto palatino mayor.

\begin{tabular}{|c|c|c|c|c|c|}
\hline Rel Se & =PM/PD & \multicolumn{2}{|c|}{ Permeabilidad CPM } & \multicolumn{2}{|r|}{ Relacion FPM/CPM } \\
\hline \multicolumn{6}{|c|}{ Lado Derecho } \\
\hline Canino & $15(48,39 \%)$ & $\mathrm{Si}$ & $31(100 \%)$ & $\mathrm{Si}$ & $22(70,97 \%)$ \\
\hline Entre C/PM & $2(6,45 \%)$ & No & 0 & No & $9(29,03 \%)$ \\
\hline PM & $14(45,16 \%)$ & Parcial & 0 & & \\
\hline \multicolumn{6}{|c|}{ Lado Izquierdo } \\
\hline Canino & $14(45,16 \%)$ & $\mathrm{Si}$ & $31(100 \%)$ & $\mathrm{Si}$ & $21(67,74 \%)$ \\
\hline Entre C/PM & $1(3,23 \%)$ & No & 0 & No & $10(32,26 \%)$ \\
\hline PM & $16(51,61 \%)$ & Parcial & 0 & & \\
\hline
\end{tabular}

SALCEDO, A.; ARAYA, C.; SILVA, J.; BARRAZA, N. \& LATÍN, A. Contribution to the descriptive study of the foramen and greater palatine canal. Int. J. Odontostomat. 13(1):40-45, 2019.

ABSTRACT: The greater palatine foramen and canal (GPF and GPC) communicate with the pterygopalatine fossa. The adequate knowledge of its morphology allows the anesthetic approach of the maxillary nerve. In vivo, the GPF is covered by a thick mucosa, therefore, the bone and dental reference points are important to locate the puncture site. Great variability has been described in terms of ethnicity, position, shape, diameters, lengths and permeability. The objective of this study was to describe these characteristics 
in skulls of Chilean adults. 31 skulls of both sexes were used. In the palates shape, length, depth and width were determined. In the GPF its shape, diameters and location were considered. In the GPC, their permeability and their coincidence with the shape of the GPF were recorded. The records were made with digital camera, precision compass, digital caliper, Korkhaus three-dimensional compass and metallic probe. The results show a predominance of the square shape of the palate over the triangular and rounded forms. The measurements of its length, width and depth indicate differences by sex and ethnicity. The shape of the GPF shows no difference by sex, with the oval shape prevailing over the fusiform and the rounded. The position of this same foramen also shows no sexual differences, with the position prevailing against the upper third molar, followed by the position in front of the space between the upper second and third molars and finally against the upper 2-molar. The dimensions of GPF are greater in male individuals. The GPC were found to be entirely permeable and the GPF did not always coincide in form with the cross section of the GPC. These results and their comparison with the literature indicate important variations, which prevents establishing objective guidelines for the anesthetic technique used in these cases.

KEY WORDS: greater palatine foramen, greater palatine canal, pterygopalatine fossa, maxillary bone, palatal bone, Carrea anesthetic technique.

\section{REFERENCIAS BIBLIOGRÁFICAS}

Ajmani, M. L. Anatomical variation in position of the greater palatine foramen in the adult human skull. J. Anat. 184(Pt. 3):635-7, 1994.

Alvarado, M.; Canala-Echeverria, M.; D’Acuña, E.; Gonzalez, D.; Mejias, R.; Oyangurem, P.; Quezada, P. \& Rodriguez, S. Anatomía Aplicada al Sistema Estomatognático. Santiago de Chile, Ediciones Universidad de Chile, 1982.

Aprile, H.; Figún, M. \& Garino, R. Anatomía Odontológica Orocervicofacial. $4^{\mathrm{a}}$ ed. Buenos Aires, El Ateneo, 1967.

Beaunis, H. \& Bouchard, A. Nuevos Elementos de Anatomía Descriptiva y de Embriología. $2^{\text {a }}$ ed. Madrid, Moya y Plaza, Libreros Editores, 1878.

Carrea, J. U. Anestesia troncular del nervio maxilar superior por el conducto palatino posterior. Odontologia, 266-71, 1921.

Cruveilhier, J. Traité d'Anatomie Descriptive. Paris, Labé, 1851.

Dauber, W. Feneis. Nomenclatura Anatómica Ilustrada. $5^{\mathrm{a}}$ ed. Barcelona, Masson, 2007.

Howard-Swirzinski, K.; Edwards, P. C.; Saini, T. S. \& Norton, N. S. Length and geometric patterns of the greater palatine canal observed in cone beam computed tomography. Int. J. Dent., 2010:292753, 2010.

Ilayperuma, I.; Nanayakkara, G. \& Palahepitiya, N. Morphometric evaluation of the greater palatine foramen in adult Sri Lankan skulls. Int. J. Morphol., 32(4):1418-22, 2014.

Latarjet, M. \& Ruiz Liard, A. Anatomía Humana. Buenos Aires, Médica Panamericana, 2010.

Lopes, P. T. C.; Santos, A. M. P. V.; Pereira, G. A. M. \& Oliveira, V. C. B. D. Morphometric analysis of the greater palatine foramen in dry southern Brazilian adult skulls. Int. J. Morphol., 29(2):420-3, 2011.
Malamed, S. F. \& Trieger, N. Intraoral maxillary nerve block: an anatomical and clinical study. Anesth. Prog., 30(2):44-8, 1983.

Malamed, S. Manual de Anestesiología Local. $5^{\mathrm{a}}$ ed. Barcelona, Elsevier, 2006.

Methathrathip, D.; Apinhasmit, W.; Chompoopong, S.; Lertsirithong, A.; Ariyawatkul, T. \& Sangvichien, S. Anatomy of greater palatine foramen and canal and pterygopalatine fossa in Thais: considerations for maxillary nerve block. Surg. Radiol. Anat., 27(6):511-6, 2005.

Nimigean, V.; Nimigean, V. R.; Butincu, L.; Salavastru, D. I. \& Podoleanu, L. Anatomical and clinical considerations regarding the greater palatine foramen. Rom. J. Morphol. Embryol., 54(3 Suppl.):779-83, 2013.

Poirier, P.; Charpy, A. \& Cuneo, B. Tratado Elemental de Anatomía Humana. Madrid, Imprenta y Librería de Nicolas Moya, 1906.

Rapado-González, O.; Suárez-Quintanilla, J. A.; Otero-Cepeda, X. L.; Fernández-Alonso, A. \& Suárez-Cunqueiro, M. M. Morphometric study of the greater palatine canal: cone-beam computed tomography. Surg. Radiol. Anat., 37(10):1217-24, 2015.

Ries-Centeno, G. Cirugía Bucal. $7^{\mathrm{a}}$ ed. Buenos Aires, El Ateneo, 1973.

Sappey, P. Tratado de Anatomía Descriptiva. Madrid, Carlos BaillyBailliere, 1874.

Sarilita, E. \& Soames, R. Morphology of the hard palate: a study of dry skulls and review of the literature. Rev. Argent. Anat. Clin., 7(1):34-43, 2015.

Sheikhi, M.; Zamaninaser, A. \& Jalalian, F. Length and anatomic routes of the greater palatine canal as observed by cone beam computed tomography. Dent. Res. J. (Isfahan), 10(2):155-61, 2013.

Sicher, H. \& Tandler, J. Anatomía para Dentistas. Barcelona, Labor, 1930.

Testut, L. \& Latarjet, A. Tratado de Anatomía Humana. Barcelona, Salvat Editores, 1965.

Tomaszewska, I. M.; Kmiotek, E. K.; Pena, I. Z.; Sredniawa, M.; Czyzowska, K.; Chrzan, R.; Nowakowski, M. \& Walocha, J. A. Computed tomography morphometric analysis of the greater palatine canal: a study of 1,500 head CT scans and a systematic review of literature. Anat. Sci. Int., 90(4):287-97, 2015.

Tomaszewska, I. M.; Tomaszewski, K. A.; Kmiotek, E. K.; Pena, I. Z.; Urbanik, A.; Nowakowski, M. \& Walocha, J. A. Anatomical landmarks for the localization of the greater palatine foramen--a study of 1200 head CTs, 150 dry skulls, systematic review of literature and meta-analysis. J. Anat., 225(4):419-35, 2014.

Triantafilo, P. La Cavidad Oral Vista por el Odontólogo. Santiago, Ediciones Universidad de Chile, 1968.

Velayos, J. Anatomía de la Cabeza. $3^{\mathrm{a}}$ ed. Madrid, Panamericana, 2001.

Dirección para correspondencia:

Dr. Andrés Salcedo G.

Facultad de Odontología

Universidad San Sebastian

Bellavista 7, Recoleta

Santiago - CHILE

Email: asalcedog@docente.uss.cl

Recibido : 30-07-2018

Aceptado: $17-10-2018$ 